\title{
ОПРЕДЕЛЕНИЕ ПОЛИСУЛЬФИД-ИОНОВ В ЩЕЛОЧНОЙ СРЕДЕ В УСЛОВИЯХ КАТОДНОЙ ИНВЕРСИОННОЙ ВОЛЬТАМПЕРОМЕТРИИ С РТУТНО-ПЛЕНОЧНЫМ ЭЛЕКТРОДОМ
}

\author{
Ковалева Светлана Владимировна ${ }^{1}$, \\ svetkovaleva@rambler.ru
}

\author{
Коршунов Андрей Владимирович2, \\ korshunov@tpu.ru
}

\section{Аксиненко Ольга Сергеевна ${ }^{3}$, crumba88@mail.ru}

\author{
Абрамова Полина Владимировна², \\ polya.bozhcko@yandex.ru \\ 1 Томский государственный педагогический университет, \\ Россия, 634061 г. Томск, ул. Киевская, 60. \\ 2 Национальный исследовательский Томский политехнический университет, \\ Россия, 634050 г. Томск, пр. Ленина, 30. \\ 3 Управление образования Администрации Томского района, \\ Россия, 634009, г. Томск, пер. Кооперативный, 2а.
}

\begin{abstract}
Актуальность. Полисульфиды щелочных, щелочноземельных металлов и аммония являются компонентами технологических сред в крупнотоннажных производствах (щёлоки в технологии челлюлозы; растворы чернения и воронения; реагенты для производства полисульфидных эластомеров), используются для получения новых функциональных материалов (герметики), играют определяющую роль при функционировании химических источников тока нового поколения. В связи с этим изучение закономерностей протекания электродных процессов с участием полисульфидов является актуальным.

Цель: установить условия определения полисульфид-ионов в щелочных растворах с использованием катодной инверсионной вольтамперометрии с ртутно-пленочным электродом.

Объекты: растворы полисульфридов натрия $\mathrm{Na}_{2} \mathrm{~S}_{2}$ и $\mathrm{Na}_{2} \mathrm{~S}_{3}$, растворы гидроксида натрия.

Методы: постоянно- и переменнотоковая катодная инверсионная вольтамперометрия, циклическая вольтамперометрия, накопительный электролиз.

Результаты. Предложены эмпирические уравнения для расчета изменения энергии Гиббса образования $S_{n}^{2-}-$-ионов в водных растворах и их стандартных потенциалов в зависимости от степени полисульфидности в интервале $n=1$...8. Проведены расчеты равновесных активностей ионно-молекулярных форм в системе $\mathrm{Hg}-\mathrm{S}-\mathrm{H}_{2} \mathrm{O}$ при различных значениях рН и потенциалов. Установлены оптимальные условия определения полисульфид-ионов $\mathrm{S}_{2}{ }^{2-}$ u $\mathrm{S}_{3}{ }^{2-}$ в щелочной среде $(0,1 \mathrm{M} \mathrm{NaOH})$ на pmymно-пленочном электроде с использованием постоянно- и переменнотоковой катодной инверсионной вольтамперометрии: потенциал предэлектролиза -0,5 B (х. с. э.); продолжительность предэлектролиза 1 ...2 мин; последующая катодная поляризация до $E_{к о н}=-1,0$ B; предварительное деаэрирование растворов азотом. $B$ этих условиях концентрационная зависимость величины максимума катодного тока $I_{k}$ при $E_{\max }=-0,8 \ldots-0,9$ В линейна в интервале концентраций полисульфридов $1 \cdot 10^{-7} . .1 \cdot 10^{-3}$ M. Метод может быть использован для определения общего содержания полисульфидов в растворе и для расчета средней степени полисульфридности в ионах $\mathrm{S}_{n}^{2-}$.
\end{abstract}

\section{Ключевые слова:}

Полисульфид-ионы, щелочные электролиты, постоянно- и переменнотоковая катодная инверсионная вольтамперометрия, циклическая вольтамперометрия, ртутно-пленочный электрод, катодный процесс.

\section{Введение}

Полисульфиды щелочных, щелочноземельных металлов и аммония являются компонентами технологических сред в крупнотоннажных производствах (щёлоки в технологии целлюлозы [1]; растворы чернения и воронения [2]; реагенты для производства полисульфидных эластомеров [3]), используются для получения новых функциональных материалов (герметики [4], играют определяющую роль при функционировании химических источников тока нового поколения $[5,6]$. В последнее время появились новые данные об участии полисульфидов в метаболических процессах [7]. Несмотря на достаточно длительный период изучения полисульфидов, оптимальные мето- ды их определения в растворах находятся на стадии разработки [8]. Причиной этого, прежде всего, является совместное присутствие практически всех возможных форм $\left(\mathrm{S}_{2}{ }^{2-}-\mathrm{S}_{8}{ }^{2-}\right.$ в водных растворах $)$ в равновесных смесях: характеристики отдельных форм мало отличаются друг от друга, что не позволяет дифференцировать аналитический сигнал от каждой из них. Кроме того, эти соединения малоустойчивы, вследствие чего не исключена вероятность их разложения в ходе анализа [9]. В связи с этим, изучение полисульфидных систем является актуальным как в фундаментальном, так и в прикладном аспектах.

Известны методы определения полисульфидов в растворах с использованием спектрофотометрии, 
хроматографии, ЯМР-спектроскопии, фотоэлектронной спектроскопии, а также электроаналитические методы [9]. Основная трудность спектральных и хроматографических методов определения индивидуальных полисульфидов связана с дифференцированием аналитического сигнала от различных форм $\mathrm{S}_{n}{ }^{2-}[10]$. В ряде объектов (морская вода) целесообразно определять совокупность восстановленных форм серы $\left(\mathrm{S}_{2} \mathrm{O}_{3}{ }^{2-}+\mathrm{SO}_{3}{ }^{2-} ; \mathrm{S}^{0}+\mathrm{S}_{n}{ }^{2-}\right)$ с использованием осаждения в виде серы и малорастворимых соединений $(\mathrm{ZnS})$ и последующим их раздельным спектрофотометрическим определением [11].

Наиболее селективным методом определения отдельных форм $\mathrm{S}_{n}{ }^{2-}$ является метод жидкостной хромато-масс-спектрометрии продуктов взаимодействия полисульфидов с 4-(диметиламино)бензоилхлорилом в неводной среде (диметоксиэтан, ацетонитрил) в атмосфере аргона [12]. Для завершения реакции между полисульфидами и дифференцирующим реагентом необходимо выдерживать реакционную смесь в течение 2 ч в инертной атмосфере. Из результатов анализа модельной смеси следует, что в синтезированном растворе $\mathrm{Na}_{2} \mathrm{~S}_{4}$ присутствовали все формы $\mathrm{S}_{2}{ }^{2-}-\mathrm{S}_{8}{ }^{2-}$, наибольшее содержание достигалось для $\mathrm{S}_{3}{ }^{2-}$, в $3 . .4$ раза меньшие количества приходились на $\mathrm{S}_{2}{ }^{2-}$, и $\mathrm{S}_{4}{ }^{2-}-\mathrm{S}_{6}{ }^{2-}$ [12]. Терминальные и центральные атомы серы в полимерных формах $\mathrm{S}_{n}{ }^{2-}$ в составе твердых образцов могут быть идентифицированы при помощи рентгеновской фотоэлектронной спектроскопии [13]. Методом ЯМР-спектроскопии показано, что в щелочных растворах полисульфидов совместно присутствуют формы $\mathrm{S}_{2}{ }^{2-}-\mathrm{S}_{6}{ }^{2-}[14]$.

Электрохимические методы определения $\mathrm{S}_{n}{ }^{2-}$ являются более простыми по аппаратурному оформлению, числу операций, затрачиваемому времени и используемым реактивам. В работе [15] предложен метод вольтамперометрического определение алкильных производных полисульфидов в среде ацетонитрила на Pt-электроде. Показано, что при анодной поляризации электрода на вольтамперограммах присутствуют максимумы токов окисления в интервале $1 \ldots 2$ В (н. к. э.), соответствующие дисульфиду $(1,3$ В), трисульфиду (1,7 В) и тетрасульфиду (1,9 В). В соответствии с полученными данными, при приготовлении растворов различные формы полисульфидов образуются параллельно, большая доля приходится на трисульфид; тетрасульфид и дисульфид образуются в меньших количествах. Нужно отметить, что независимая проверка результатов вольтамперометрического анализа с использованием жидкостной хроматографии и ЯМР-спектроскопии [15] показала низкую сходимость полученных данных. В ряде работ показана возможность определения различных форм серы в природных водах вольтамперометрическом методом по величине токов их окисления или восстановления, в том числе в форме $\mathrm{HgS}[16,17]$.

Катодное восстановление $\mathrm{S}_{2}{ }^{2-}$-ионов на ртутном капающем электроде при $\mathrm{pH}>9$ протекает в области потенциалов $-0,8$ В (н. к. э.), величина предельного тока линейно зависит от концентрации деполяризато- pa [18]. Йодометрический анализ продуктов восстановления в контролируемых условиях электролиза и кулонометрический анализ ( $c=1,26 \mathrm{MM} \mathrm{S}_{2}{ }^{2-}, \tau=3$ ч, $q=2,30$ мКл) показали, что восстановлению подверглось $1,19 \mathrm{MM} \mathrm{S}_{2}{ }^{2-}$-ионов, при этом образовалось

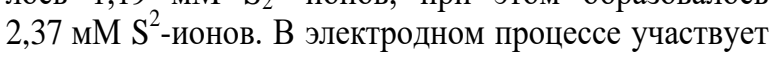
2 электрона, что соответствует электродной реакции [18]:

$$
\mathrm{S}_{2}^{2-}+2 \overline{\mathrm{e}} \rightarrow 2 \mathrm{~S}^{2-} .
$$

Восстановление $\mathrm{S}_{4}{ }^{2-}$-ионов отображается на полярограммах в виде аналогичной необратимой волны, предельный ток достигается при $-1,80 \mathrm{~B}$, и его величина линейно зависит от концентрации $\mathrm{S}_{4}{ }^{2-}$-ионов [19]. Подкисление растворов полисульфидов приводит к уменьшению величины катодного тока вследствие их разложения с образованием серы. Соотношение пропущенного количества электричества к количеству полисульфида составляло 5,97 F/моль, что позволило предположить протекание электродной реакции [19]:

$$
\mathrm{S}_{4}^{2-}+6 \overline{\mathrm{e}} \rightarrow 4 \mathrm{~S}^{2-} .
$$

Для $\mathrm{S}_{2}{ }^{2-}$ - и $\mathrm{S}_{4}{ }^{2-}$-ионов константы скорости и коэффициенты переноса в указанных выше условиях составили $1,9 \cdot 10^{-2}$ и $0,7 \cdot 10^{-2} \mathrm{~cm} / \mathrm{c}, 0,116$ и 0,045 , соответственно. При значении стехиометрического фактора $v=3$ для тетрасульфид-ионов $k^{0}=2,1 \cdot 10^{-2} \mathrm{~cm} / \mathrm{c}, \alpha=0,141$. На основании этих данных процесс восстановления $\mathrm{S}_{4}{ }^{2-}$-ионов может быть представлен в виде схем, включающих диспропорционирование с уменьшением степени полисульфидности [19]:

$$
\begin{array}{ll}
\mathrm{S}_{4}^{2-} \rightarrow \mathrm{S}_{3}^{2-}+\mathrm{S} & \mathrm{S}+2 \overline{\mathrm{e}} \rightarrow \mathrm{S}^{2-} \\
\mathrm{S}_{3}^{2-} \rightarrow \mathrm{S}_{2}^{2-}+\mathrm{S} & \mathrm{S}+2 \overline{\mathrm{e}} \rightarrow \mathrm{S}^{2-}
\end{array}
$$

В условиях циклической вольтамперометрии при высоких скоростях развертки потенциалов (до $1500 \mathrm{mB} / \mathrm{c}$ ) электродные процессы с участием тетрасульфида отображаются в виде двух максимумов тока [20]:

$$
\begin{aligned}
& \mathrm{S}_{4}^{2-}+\mathrm{Hg} \rightarrow \mathrm{HgS}_{4}^{0}+2 \bar{e}, \\
& \mathrm{HgS}_{4}^{0}+2 \bar{e} \rightarrow \mathrm{Hg}+\mathrm{S}_{4}^{2-}, \\
& \mathrm{S}_{4}^{2-}+4 \mathrm{H}^{+}+6 \bar{e} \rightarrow 4 \mathrm{HS}^{-} .
\end{aligned}
$$

Вследствие большей энергии связи в $\mathrm{HgS}_{4}$, чем в моносульфиде, восстановление полисульфида протекает при более отрицательных потенциалах. Cера $\mathrm{S}^{0}$ в составе полисульфида восстанавливается при еще более отрицательных потенциалах. При низких скоростях развертки потенциала $(25 \ldots 75 \mathrm{MB} / \mathrm{c})$ электродный процесс с участием $\mathrm{HS}^{-}$-ионов является обратимым [20].

В условиях дифференциально-импульсной полярографии анодное окисление полисульфидов в присутствии политионатов и сульфидов $(\mathrm{pH}=9,5)$ можно представить в виде схем [21]:

$$
\begin{gathered}
\mathrm{S}_{n}^{2-}+\mathrm{Hg} \rightarrow \mathrm{HgS}+(n-1) \mathrm{S}+2 \bar{e} . E_{1 / 2}=-0,68 \mathrm{~B} ; \\
\mathrm{HS}^{-}+\mathrm{Hg} \rightarrow \mathrm{HgS}+\mathrm{H}^{+}+2 \bar{e}, E_{1 / 2}=-0,68 \mathrm{~B} .
\end{gathered}
$$


При эквимолярных концентрациях сульфид- и дисульфид-ионов анодный ток на полярограммах одинаковый. Восстановление полисульфид-ионов протекает по схеме [21]:

$$
\mathrm{S}_{n}^{2-}+(2 n-1) \bar{e}+n \mathrm{H}_{2} \mathrm{O} \rightarrow n \mathrm{HS}^{-}+n \mathrm{OH}^{-}, E_{1 / 2}=-0,60 \mathrm{~B} .
$$

Метод дифференциально-импульсной полярографии пригоден для определения $\mathrm{S}^{0}$ в интервале $10^{-5}-10^{-3} \mathrm{M}$. Тиосульфат и политионаты не оказывают мешающего влияния при определении полисульфидов.

Из анализа литературных данных по методам определения полисульфидов в растворах следует, что применительно к технологическим средам и объектам окружающей среды использование многостадийной процедуры и дорогостоящего оборудования для раздельного определения отдельных форм $\mathrm{S}_{\mathrm{n}}{ }^{2-}$ нецелесообразно. Такие формы находятся в состоянии равновесия, и максимальная доля приходится на ионы $\mathrm{S}_{3}{ }^{2-}-\mathrm{S}_{5}{ }^{2-}$, с позиции аналитической практики достаточно определить общее содержание полисульфидов и рассчитать среднюю степень полисульфидности. С учетом этого наиболее целесообразным является использование в качестве аналитического сигнала тока восстановления $\mathrm{HgS}$ по аналогии с методом определения сульфид-ионов [22, 23]. Целью работы являлось установление условий определения полисульфид-ионов в щелочных растворах с использованием катодной инверсионной вольтамперометрии с ртутным пленочным электродом.

\section{Материалы и методы исследования}

Электрохимическое поведение полисульфидов в растворах изучали с использованием методов постоянно- и переменнотоковой вольтамперометрии (полярограф ПУ-1 с двухкоординатным графопостроителем Н307). Измерения проводили с использованием трехэлектродной ячейки с разделенным электродным пространством. В качестве рабочего электрода использовали ртутно-пленочный электрод (РПЭ), представляющий собой серебряную проволоку длиной 7 мм и диаметром 1 мм, запрессованную в полимерный стержень. Площадь рабочей поверхности электрода составляла $0,22 \mathrm{~cm}^{2}$, на нее наносили тонкую пленку ртути путем погружения в металлическую ртуть. Готовый электрод хранили в бидистиллированной воде. Вспомогательным электродом являлся графитовый стержень площадью $3,69 \mathrm{~cm}^{2}$, электродом сравнения служил хлорсерябряный электрод, заполненный насыщенным раствором $\mathrm{KCl}$ (х. с. э.). Потенциалы в работе приведены относительно х. с. э.

Приготовление растворов полисульфидов проводили следующим образом. Исходным раствором служил раствор $0,1 \mathrm{M} \mathrm{Na} 2 \mathrm{~S}$, который готовили растворением навесок $\mathrm{Na}_{2} \mathrm{~S} \cdot 9 \mathrm{H}_{2} \mathrm{O}$ в деаэрированной воде. Точность концентрации приготовленного раствора определяли методом обратного йодометрического титрования [24]. Получение растворов полисульфидов осуществляли в соответствии с уравнением реакции:

$$
\mathrm{Na}_{2} \mathrm{~S}+(n-1) \mathrm{S} \rightarrow \mathrm{Na}_{2} \mathrm{~S}_{n} .
$$

Для этого к 20 мл 0,1 M раствора $\mathrm{Na}_{2} \mathrm{~S}$ добавляли рассчитанную по уравнению реакции навеску ромбической серы и нагревали раствор при температуре $60{ }^{\circ} \mathrm{C}$ до полного растворения элементной серы. Определение концентрации полисульфид-ионов проводили методом йодометрии. Йод в кислой среде взаимодействует с полисульфидами в соответствии с уравнениями:

$$
\begin{gathered}
\mathrm{Na}_{2} \mathrm{~S}_{n}+\mathrm{H}_{2} \mathrm{SO}_{4}=\mathrm{H}_{2} \mathrm{~S}_{n}+\mathrm{Na}_{2} \mathrm{SO}_{4}, \\
\mathrm{H}_{2} \mathrm{~S}_{n}+\mathrm{I}_{2}=2 \mathrm{HI}+n \mathrm{~S} .
\end{gathered}
$$

Расчет концентрации ионов $\mathrm{S}_{\mathrm{n}}{ }^{2-}$ проводили по формулам:

$$
c_{\mathrm{H}}\left(\mathrm{S}_{n}^{2-}\right)=\frac{c_{\mathrm{H}}\left(\mathrm{I}_{2}\right) V\left(\mathrm{I}_{2}\right)}{V\left(\mathrm{~S}_{n}^{2-}\right)}, c_{\mathrm{M}}\left(\mathrm{S}_{n}^{2-}\right)=\frac{1}{2} c_{\mathrm{H}}\left(\mathrm{S}_{n}^{2-}\right),
$$

где $c_{\mathrm{H}}$ и $c_{\mathrm{M}}-$ нормальная и молярная концентрации, моль/л; $V$ - объем раствора, мл. Концентрацию приготовленных растворов $\mathrm{Na}_{2} \mathrm{~S}_{2}$ и $\mathrm{Na}_{2} \mathrm{~S}_{3}$ контролировали методом йодометрического титрования (табл. 1).

Таблица 1. Результаты определения конщентрации полисульфидов в растворах, полученных для

\begin{tabular}{|c|c|c|c|}
\hline $\begin{array}{l}\text { Формула } \\
\text { Formula }\end{array}$ & $\mid \begin{array}{l}m(\mathrm{~S}), \Gamma \\
m(\mathrm{~S}), \mathrm{g}\end{array}$ & $\begin{array}{c}\text { Заданная } \\
\text { концентрация } \\
\mathrm{Na}_{2} \mathrm{~S}_{n}, \mathrm{M} \\
\text { Preset } \\
\text { concentration } \\
\text { of } \mathrm{Na}_{2} \mathrm{~S}_{n}, \mathrm{M} \\
\end{array}$ & $\begin{array}{c}\text { Концентрация } \mathrm{Na}_{2} \mathrm{~S}_{\mathrm{n}} \text { по } \\
\text { данным йодометрического } \\
\text { титрования, } \mathrm{M} \\
\mathrm{Na}_{2} \mathrm{~S}_{\mathrm{n}} \text { concentration } \\
\text { determined by iodometric } \\
\text { titration, } \mathrm{M}\end{array}$ \\
\hline $\mathrm{Na}_{2} \mathrm{~S}_{2}$ & 0,064 & $2 \cdot 10^{-2}$ & $1,8 \cdot 10^{-2}$ \\
\hline $\mathrm{Na}_{2} \mathrm{~S}_{3}$ & 0,128 & $2 \cdot 10^{-2}$ & $1,9 \cdot 10^{-2}$ \\
\hline
\end{tabular}
проведения электрохимических измерений

Table 1. Results of determination of polysulfides concentration in solutions prepared for electrochemical experiments

Для приготовления вспомогательных и фоновых растворов ( $\mathrm{KCl}, \mathrm{NaOH}, \mathrm{Na}_{2} \mathrm{~S}_{2} \mathrm{O}_{3}, \mathrm{I}_{2}$, крахмал) использовали реактивы и фиксаналы квалификации «х. ч.» и «ч. д. а.» без дополнительной очистки. Растворы готовили с использованием бидистиллированной воды. Деаэрирование растворов в ходе электрохимических измерений проводили путем пропускания через них азота высокой чистоты.

\section{Результаты и их обсуждение}

Для уточнения значений стандартных потенциалов полисульфидных систем использовали метод расчета, основанный на термодинамических характеристиках ионов в растворах. В табл. 2 приведены величины изменения энергий Гиббса и стандартные потенциалы моно- и полисульфид-ионов из литературных источников [25-29].

Термодинамические характеристики моно- и полисульфид-ионов в [25] определены методом измерения редокс-потенциалов систем сера-полисульфидион. Авторы работы [26] рассчитывали термодинамические функции по уравнению:

$$
\Delta G_{298}^{0}\left(\mathrm{~S}_{n}{ }^{2-}\right)=\Delta G_{298}^{0}\left(\mathrm{~S}^{2-}\right)+(n-1) \Delta G_{298}^{0}\left(\mathrm{~S}^{2-}\right),
$$

где $\Delta G_{298}^{0}$ - изменение энергии Гиббса образования вещества, $n$ - степень полисульфидности. В работе 
[27] на основании предположения об аддитивности термодинамических величин в ряду моносульфид полисульфид-анион была предложена эмпирическая зависимость $\Delta G_{298}^{0}$ реакций генерации $\mathrm{S}_{n}{ }^{2-}$-анионов от числа атомов серы в анионе и заряда $z$ :

$$
\Delta G_{298}^{\mathrm{o}}\left(\mathrm{S}_{n}{ }^{2-}\right)=\Delta G_{298}^{\mathrm{o}}\left(\mathrm{S}^{2-}\right)+(\mathrm{n}-1) \Delta G_{298}^{\mathrm{o}}\left(\mathrm{S}^{2-}\right) / 6 z .
$$

Таблица 2. Величины изменения энергии Гиббса и стандартные потенциалы для моно- и полисульфид-ионов в водных растворах

Table 2. The Gibbs energies and standard potentials for mono- and polysulfide ions in aqueous solutions

\begin{tabular}{|c|c|c|c|c|c|}
\hline \multirow{2}{*}{$\begin{array}{c}\text { Ион } \\
\text { Ion }\end{array}$} & {$[25]$} & {$[26]$} & {$[27]$} & {$[28]$} & {$[29]$} \\
\hline $\mathrm{S}^{2-}$ & $91,87 / 0,476$ & $91,87 / 0,476$ & $91,87 / 0,476$ & $85,77 / 0,444$ & $91,87 / 0,476$ \\
\hline $\mathrm{S}_{2}{ }^{2-}$ & $82,63 / 0,428$ & $82,30 / 0,427$ & $84,21 / 0,436$ & $79,50 / 0,412$ & $82,68 / 0,428$ \\
\hline $\mathrm{S}_{3}{ }^{2-}$ & $75,18 / 0,390$ & $72,81 / 0,378$ & $76,56 / 0,397$ & $73,64 / 0,382$ & $75,33 / 0,390$ \\
\hline $\mathrm{S}_{4}{ }^{2-}$ & $69,52 / 0,360$ & $63,32 / 0,328$ & $68,90 / 0,357$ & $69,04 / 0,358$ & $69,82 / 0,362$ \\
\hline $\mathrm{S}_{5}{ }^{2-}$ & $65,64 / 0,340$ & $54,26 / 0,280$ & $61,25 / 0,317$ & $65,69 / 0,340$ & $66,15 / 0,343$ \\
\hline $\mathrm{S}_{6}{ }^{2-}$ & - & $44,81 / 0,230$ & $53,59 / 0,278$ & - & $64,31 / 0,333$ \\
\hline
\end{tabular}

Анализ данных табл. 2 показал, что зависимость $\Delta G^{\mathrm{o}}{ }_{298}\left(\mathrm{~S}_{n}{ }^{2-}\right)$ от степени полисульфидности носит экспоненциальный характер. Вид этой зависимости аналогичен зависимости удельного заряда атомов $\mathrm{S}$ от их числа в анионе. Как и в случае удельного заряда, разница в величинах $\Delta G_{298}^{0}$ соседних анионов максимальна для первых двух членов гомологического ряда, при переходе к последующим анионам она постепенно уменьшается. В связи с этим было предложено новое эмпирическое уравнение для расчета $\Delta G_{298}^{0}$ полисульфид-ионов [29]:

$$
\Delta G_{298}^{\mathrm{o}}\left(\mathrm{S}_{n}{ }^{2-}\right)=\Delta G_{298}^{\mathrm{o}}\left(\mathrm{S}^{2-}\right)-0,01(12-\mathrm{n})(\mathrm{n}-1) \Delta G^{\mathrm{o}}{ }_{298}\left(\mathrm{~S}^{2-}\right) .
$$

На основании полученных значений $\Delta G_{298}^{0}\left(\mathrm{~S}_{n}{ }^{2-}\right)$ были рассчитаны стандартные потенциалоы, приведенные в табл. 2. Регрессионный анализ зависимости $E^{\circ}=f(n)$ по данным табл. 2 позволил вывести эмпирическое уравнение для расчета стандартных потенциалов (табл. 3).

Таблица 3. Уравнение регрессии, описывающее зависимость $E^{\circ}$ полисульфидных систем от $n$

Table 3. Regression equation for dependence of $E^{\circ}$ on $n$

\begin{tabular}{|c|c|c|c|}
\hline $\begin{array}{c}\text { Интервал } \\
\text { значений n в } \\
\text { ионах } \mathrm{S}_{n}{ }^{2-} \\
\text { Interval of n } \\
\text { values for } \mathrm{S}_{n}{ }^{2-}\end{array}$ & $\begin{array}{c}\text { Уравнение } \\
\text { регрессии } \\
\text { Regression } \\
\text { equation }\end{array}$ & $\begin{array}{c}\text { Значения коэф- } \\
\text { фициентов } \\
\text { Coefficients }\end{array}$ & $\begin{array}{c}\text { Коэффициент } \\
\text { корреляции } \\
\mathrm{R}(P=0,95) \\
\text { Correlation } \\
\text { coefficient } \\
\mathrm{R} \text { at } P=0,95\end{array}$ \\
\hline $1 \ldots 8$ & $E^{\circ}=a \mathrm{e}^{-b n}+c \mathrm{e}^{-d n}$ & $\begin{array}{c}a=0,2508 \\
b=0,3310 \\
c=0,2967 \\
d=2,355 \cdot 10^{-10}\end{array}$ & 0,999 \\
\hline
\end{tabular}
for polysulfide systems

С использованием предложенного уравнения зависимости $E^{\circ}=f(\mathrm{n})$ произведен расчет значений стандартных потенциалов полисульфид-ионов для $n=1-8$ (рис. 1). Из приведенных графических данных (рис. 1) следует, что различия значений стандартных потенциалой для $\mathrm{S}_{n}{ }^{2-}$ при $n>6$ невелики. По-видимому, образование полисульфид-ионов с $n>8$ при восстанов- лении серы в щелочной среде маловероятно, поскольку перенос электронов на молекулы серы $\mathrm{S}_{8}$ приведет к образованию ионов $\mathrm{S}_{8}{ }^{2-}$, которые далее будут восстанавливаться до полианионов с меньшим числом n. Очевидно, что с этим связано отсутствие в литературе надежных данных по полианионам $\mathrm{S}_{n}{ }^{2-} \mathrm{c}$ $n>8$ для водных растворов.

Для проверки возможности дифференцирования аналитического сигнала от отдельных полисульфидных форм построены расчетные диаграммы зависимости активностей ионно-молекулярных форм в системе $\mathrm{S}-\mathrm{H}_{2} \mathrm{O}$ (без учета $\mathrm{SO}_{4}{ }^{2-}$ ) при различных $\mathrm{pH}$ и потенциалах (рис. 2). Из диаграмм (рис. 2) следует, такие системы относительно устойчивы в узких интервалах $\mathrm{pH}$ и потенциалов: в кислой среде полисульфаны быстро разлагаются с образованием серы и сероводорода; в щелочной среде устойчивыми являются низкомолекулярные формы. В присутствии даже малоактивных окислителей полисульфиды быстро окисляются с образованием многочисленных кислородсодержащих соединений (рис. 2).

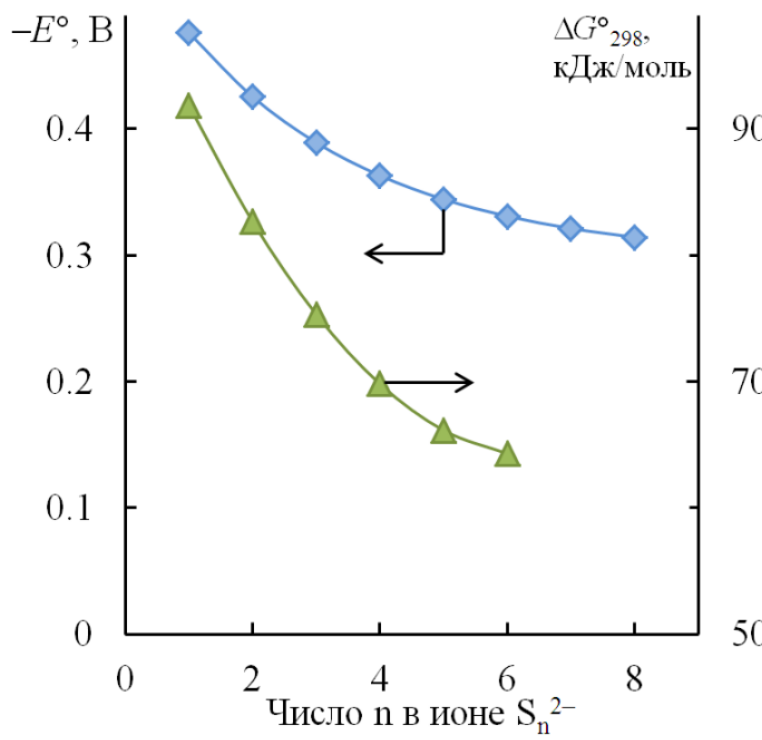

Pис. 1. Зависимости расчетных значений стандартных потенциалов $E^{\circ}$ и энергий Гиббса образования

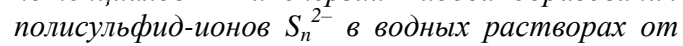
степени полисульфидности п

Fig. 1. Dependence of calculated standard potentials $E^{\circ}$ and Gibbs energies of polysulfide $S_{n}{ }^{2-}$ ions formation on the degree of polysulfidity $n$ in aqueous solutions

Наибольшее разнообразие возможных ионномолекулярных форм характерно для растворов с $\mathrm{pH}$ близким к нейтральному (рис. 2, a). Следовательно, приготовление слабощелочных водных растворов полисульфидов путем растворения стехиометрических навесок серы в сульфиде натрия приведёт к формированию равновесной системы с преобладающими формами $\mathrm{HS}^{-}, \mathrm{S}_{6}{ }^{2-}, \mathrm{S}_{5}{ }^{2-}$ и $\mathrm{S}_{4}{ }^{2-}$, причем содержание моносульфидной формы более чем на 2 порядка превышает содержание остальных форм. 
В сильнощелочной среде устойчивость полисульфидов значительно снижается, равновесное содержание преобладающих полимерных анионов $\mathrm{S}_{2}{ }^{2-}, \mathrm{S}_{3}^{2-}$ и $\mathrm{S}_{4}^{2-}$ в системе на $\sim 6$ порядков ниже по сравнению с моносульфидной формой (рис. 2, б). Очевидно, что вследствие близких значений окислительновосстановительных потенциалов полисульфидов электрохимический сигнал в таких системах является суммарным по всем присутствующим формам, необходимость его дифференциации для получения отклика отдельных компонентов для практических целей сомнительна, в особенности в условиях установления равновесия между ними.

Использование ртутного электрода для исследования процессов в растворах сульфидов приводит к изменению соотношения серосодержащих форм в растворе вследствие образования малорастворимых соединений. Вследствие высокого сродства ртути к сере преобладающей равновесной фазой в системе в широком интервале $\mathrm{pH}$ является малорастворимый $\mathrm{HgS}$, область потенциалов термодинамической устойчивости которого перекрывает область потенциалов полисульфидов (рис. 3). Соотношение различных форм полисульфидов отличается от такового в системе без ртути (рис. 2, 3), при этом активности полисульфидных форм более чем на 5 порядков меньше активности сульфида ртути. Этот эффект в большей степени проявляется для щелочной среды (рис. 3, б). Следовательно, при поляризации ртутного электрода в сульфидсодержащем растворе преобладающие по величине тока сигналы будут связаны с электродными процессами с участием $\mathrm{HgS}$, что согласуется с литературными данными [23]:

$$
\begin{aligned}
& \mathrm{Hg}+\mathrm{S}^{2-}=\mathrm{HgS}+2 \mathrm{e}, E=-0,76 \mathrm{~B} \\
& \mathrm{HgS}+2 \mathrm{e}=\mathrm{Hg}+\mathrm{S}^{2-}, E=-0,87 \mathrm{~B}
\end{aligned}
$$
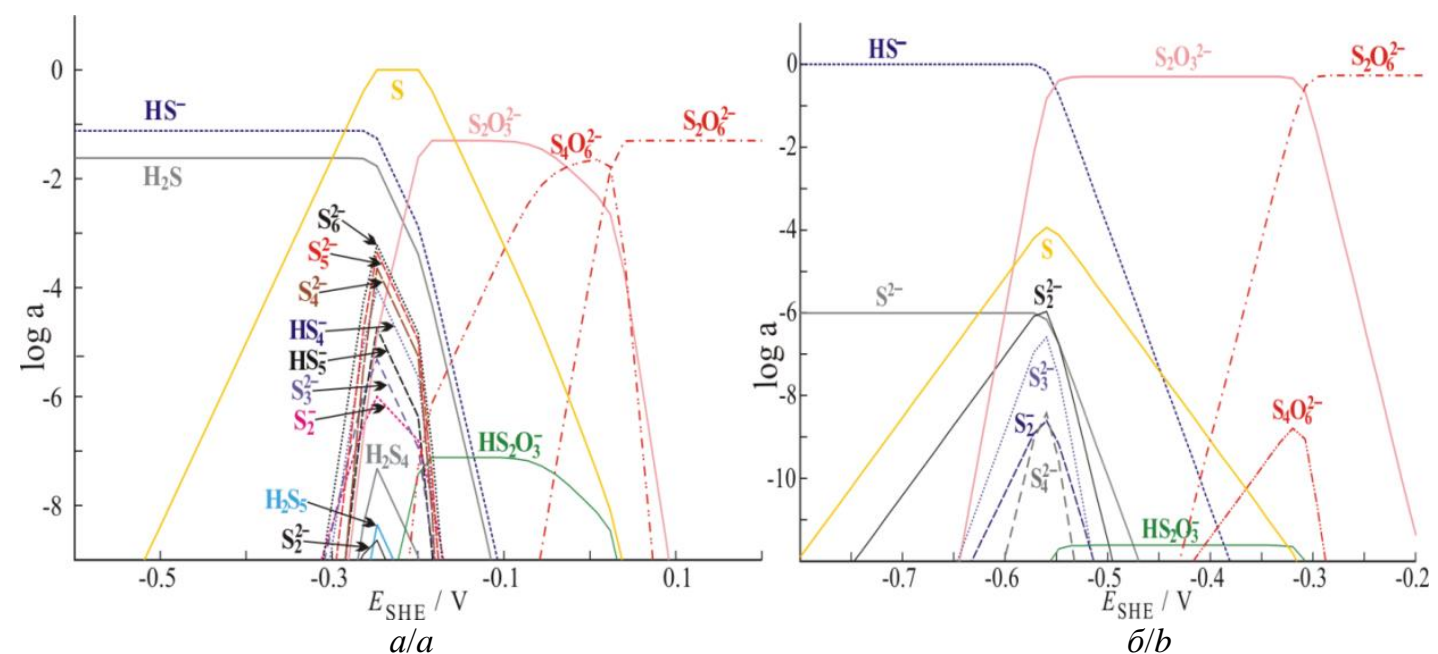

Pис. 2. Расчетные зависимости логарифмов равновесных активностей ионно-молекулярных форм от потенциала (cm. в. э.) в системе $\mathrm{S}-\mathrm{H}_{2} \mathrm{O}$ (без учета $\left.\mathrm{SO}_{4}{ }^{2-}\right)$ nри pH 7,5 (a) и 13 (б) $\left(25{ }^{\circ} \mathrm{C}\right.$; $\left.[\mathrm{S}]_{\text {обм }}=0,1 \mathrm{M}\right)$

Fig. 2. Calculated dependences of logarithms of equilibrium activities for ion-molecular species on potential (s. h. e.) for system $\mathrm{S}-\mathrm{H}_{2} \mathrm{O}$ (except $\left.\mathrm{SO}_{4}{ }^{2-}\right)$ at $\mathrm{pH} 7,5(\mathrm{a})$ and $13(\mathrm{~b})\left(25^{\circ} \mathrm{C} ;[\mathrm{S}]_{\text {tot }}=0,1 \mathrm{M}\right)$
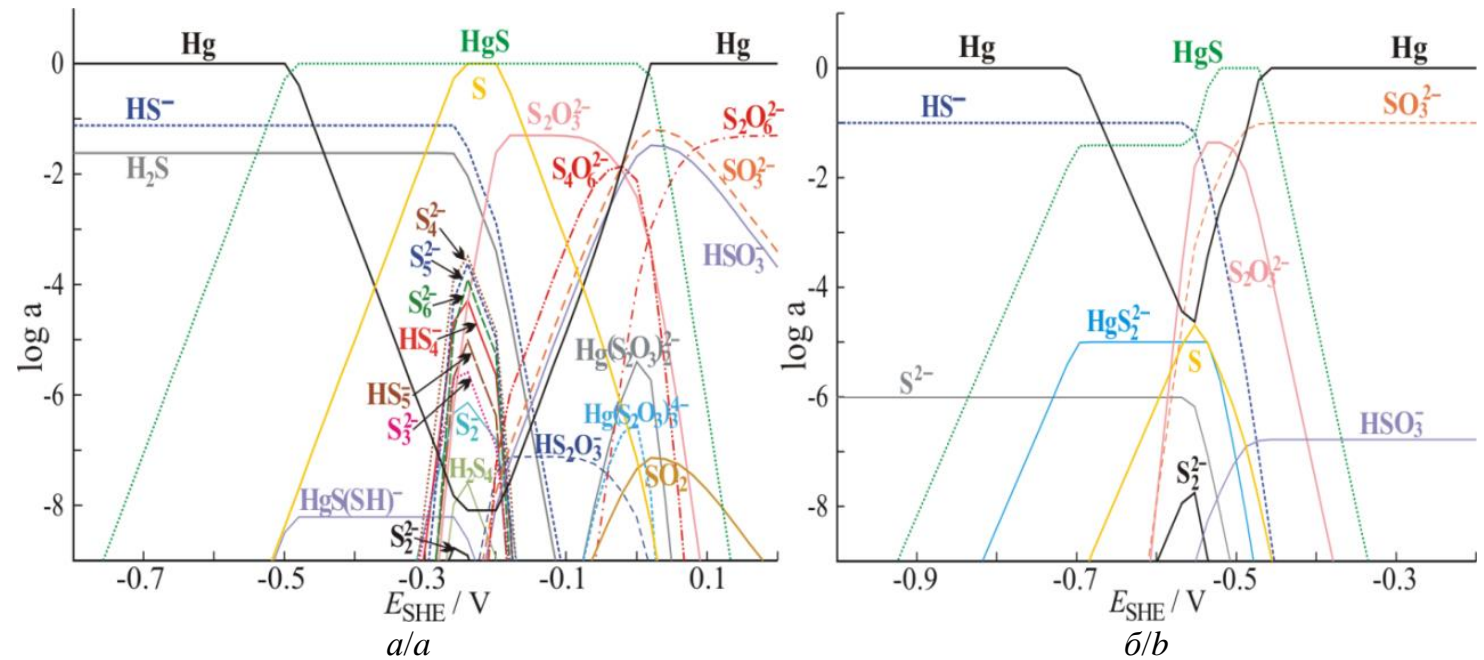

Рис. 3. Расчетные зависимости логарифмов равновесных активностей ионно-молекулярных форм от потенциала (cm. в. э.) в системе $\mathrm{Hg}-\mathrm{S}-\mathrm{H}_{2} \mathrm{O}$ (без учета $\mathrm{SO}_{4}{ }^{2-}$ ) nри $\mathrm{pH} 7,5$ (а) и 13 (б) $\left(25{ }^{\circ} \mathrm{C}\right.$; $[\mathrm{Hg}]_{\text {оби }}=1 \cdot 10^{-5} \mathrm{M}$; $\left.[\mathrm{S}]_{\text {оби }}=0,1 \mathrm{M}\right)$

Fig. 3. Calculated dependences of logarithms of equilibrium activities for ion-molecular species on potential (s. h. e.) for system $\mathrm{Hg}-\mathrm{S}-\mathrm{H}_{2} \mathrm{O}$ (except $\left.\mathrm{SO}_{4}{ }^{2-}\right)$ at $\mathrm{pH} 7,5(\mathrm{a})$ and $13(\mathrm{~b})\left(25{ }^{\circ} \mathrm{C} ;[\mathrm{Hg}]_{\text {tot }}=1 \cdot 10^{-5} \mathrm{M} ;[\mathrm{S}]_{\text {tot }}=0,1 \mathrm{M}\right)$ 
Вместе с тем при приготовлении полисульфидов путем растворения стехиометрических навесок серы в растворе $\mathrm{Na}_{2} \mathrm{~S}$ и последующего защелачивания таких растворов устойчивыми равновесными формами являются $\mathrm{S}_{2}{ }^{2-}, \mathrm{S}_{3}{ }^{2-}$ и $\mathrm{S}_{4}{ }^{2-}$ (рис. 2, б). При этом равновесные концентрации $\mathrm{S}_{2}{ }^{2-}$ и $\mathrm{S}_{3}{ }^{2-}$ при $E^{\circ} \approx-0,55 \mathrm{~B}$ (ст. в. э.) принимают близкие значения, хотя интервал потенциалов существования дисульфид-ионов значительно шире, чем для $\mathrm{S}_{3}{ }^{2-}$. Следовательно, использование сильнощелочной среды позволяет снизить число совместно присутствующих полисульфидных форм до двух основных $\mathrm{S}_{2}{ }^{2-}$ и $\mathrm{S}_{3}{ }^{2-}$, их равновесные концентрации позволяют использовать электрохимические методы для получения аналитического сигнала (рис. 2, б).
В связи с этим в экспериментах использовали растворы низших полисульфидов $\mathrm{S}_{2}{ }^{2-}$ и $\mathrm{S}_{3}{ }^{2-}$ в фоновом электролите $0,1 \mathrm{M} \mathrm{NaOH}$.

Из результатов вольтамперометрии следует, что при линейной поляризации РПЭ в растворах $\mathrm{Na}_{2} \mathrm{~S}_{2}$ в интервале $-0,5 \ldots-1,1$ В процесс восстановления протекает при $E=-0,8 \ldots-0,9$ В и отображается на вольтамперограммах в виде выраженного максимума катодного тока при $E_{\max } \approx-0,87$ В (рис. 4). Метод вольтамперометрии с РПЭ является высокочувствительным по отношению к соединениям серы, аналитический сигнал зарегистрирован в растворах $\mathrm{S}_{2}{ }^{2-}$ вплоть до концентраций $10^{-7} \mathrm{M}$ как в постоянно-, так и в переменнотоковом режиме поляризации.
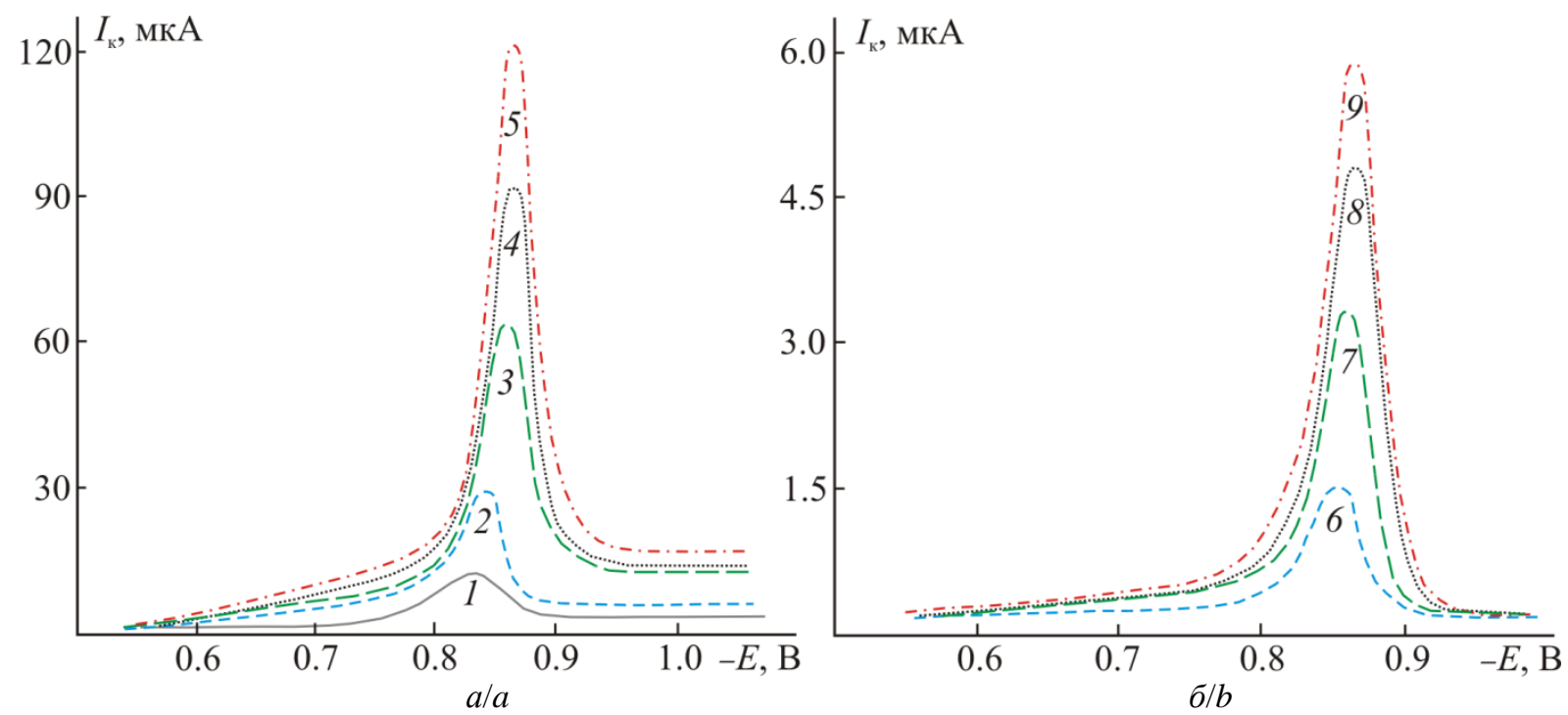

Рис. 4. Вольтамперограммы ртутно-пленочного электрода в растворах $\mathrm{Na}_{2} \mathrm{~S}_{2}+0,1 \mathrm{M} \mathrm{NaOH}$ в условиях линейной постояннотоковой (a) и переменнотоковой (б) вольтамперометрии при различных концентрациях $\mathrm{Na}_{2} \mathrm{~S}_{2}$ : 1) $4 \cdot 10^{-7}$, 2) $6 \cdot 10^{-7}$, 3) $8 \cdot 10^{-7}$, 4) $\left.1,2 \cdot 10^{-6}, 5\right) 1,6 \cdot 10^{-6}$, 6) $4 \cdot 10^{-7}$, 7) $\left.6 \cdot 10^{-7}, 8\right) 8 \cdot 10^{-7}$, 9) $1 \cdot 10^{-6} \mathrm{M}\left(E_{\text {нач }}=-0,5 \mathrm{~B}\right.$; $\left.E_{\text {кон }}=-1,1 \mathrm{~B} ; v=40 \mathrm{MB} / \mathrm{c} ; \Delta U=3 \mathrm{MB} ; \theta=0^{\circ} ; f=25 \Gamma \mathrm{u}\right)$

Fig. 4. Voltammograms of $\mathrm{Hg}$-film electrode in solutions $\mathrm{Na}_{2} \mathrm{~S}_{2}+0,1 \mathrm{M} \mathrm{NaOH}$ under conditions of direct (a) and alternating current (b) voltammetry at different concentrations of $\left.\mathrm{Na}_{2} \mathrm{~S}_{2}: 1\right) 4 \cdot 10^{-7}$, 2) $6 \cdot 10^{-7}$, 3) $\left.8 \cdot 10^{-7}, 4\right) 1.2 \cdot 10^{-6}$, 5) $1.6 \cdot 10^{-6}$, 6) $\left.\left.4 \cdot 10^{-7}, 7\right) 6 \cdot 10^{-7}, 8\right) 8 \cdot 10^{-7}$, 9) $1 \cdot 10^{-6} \mathrm{M}\left(E_{\text {init }}=-0,5 \mathrm{~V} ; E_{\text {end }}=-1,1 \mathrm{~V} ; \mathrm{v}=40 \mathrm{mV} / \mathrm{s} ; \Delta U=3 \mathrm{mV} ; \theta=0^{\circ} ; f=25 \mathrm{~Hz}\right)$

Наличие максимума катодного тока на вольтамперограммах можно объяснить следующим образом. В области потенциалов начала поляризации $E_{\text {нач }}=-0,5$ В (-0,3 В отн. ст. в. э.) происходит окисление сульфиди полисульфид-ионов на РПЭ с образованием сульфида ртути, что согласуется с расчетными (рис. 3,6 ) и литературными [23] данными:

$$
\begin{gathered}
\mathrm{S}^{2-}+\mathrm{Hg} \rightarrow \mathrm{HgS}+2 \mathrm{e}, \\
\mathrm{S}_{2}^{2-}+\mathrm{Hg} \rightarrow \mathrm{HgS}+\mathrm{S}+2 \mathrm{e} .
\end{gathered}
$$

При этом нерастворимый $\mathrm{HgS}$ накапливается на поверхности электрода и переходит в ртутную фазу с образованием амальгамы. В этом случае также возможно взаимодействие ртути с образующейся на поверхности электрода элементной серой:

$$
\mathrm{S}+\mathrm{Hg} \rightarrow \mathrm{HgS} \text {. }
$$

Протекание этих реакций приводит к модифицированию поверхности электрода пленкой сульфида ртути, который обладает электронной проводимо- стью. Это способствует параллельному окислению $\mathrm{S}_{2}{ }^{2-}$-ионов, сопровождающемуся образованием дополнительных количеств элементной серы:

$$
\mathrm{S}_{2}^{2-} \rightarrow 2 \mathrm{~S}+2 \mathrm{e} .
$$

Протекание реакций электроокисления в указанной области потенциалов с образованием $\mathrm{HgS}$ и $\mathrm{S}$ хорошо согласуется с расчетными диаграммами (рис. 3, б).

Дальнейшее смещение электродного потенциала в направлении отрицательных потенциалов приводит к восстановлению твердых фаз $\mathrm{HgS}$ и $\mathrm{S}$, которое сопровождается появлением на вольтамперограммах максимума катодного тока в области $E=-0,8 \ldots-0,9$ В (рис. 4):

$$
\begin{gathered}
\mathrm{HgS}+2 \mathrm{e} \rightarrow \mathrm{Hg}+\mathrm{S}^{2-}, \\
\mathrm{S}+2 \mathrm{e} \rightarrow \mathrm{S}^{2-} .
\end{gathered}
$$

Протекание этих процессов подтверждено регистрацией вольтамперных кривых в растворах, содержащих взвеси $\mathrm{HgS}$ и $\mathrm{S}$, при тех же условиях, что и для 
$\mathrm{S}_{2}{ }^{2-}$-ионов [30]. Кроме того, область потенциалов начала возрастания катодного тока $E<-0,7$ В (рис. 4) соответствует максимальным значениям активностей $\mathrm{HgS}$ и S на расчетных диаграммах (рис. 3, б), которые понижаются при более отрицательных потенциалах за счет восстановления и перехода в конечную ионную форму $\mathrm{S}^{2-}$. Присутствие одного выраженного максимума катодного тока в разбавленных растворах $\mathrm{Na}_{2} \mathrm{~S}_{2}$ (рис. 4) свидетельствует об определяющей роли фазы $\mathrm{HgS}$ в формировании аналитического сигнала в исследуемой системе. Отсутствие других максимумов катодного тока при более положительных или более отрицательных потенциалах подтверждает вывод о преимущественном участии одной потенциалопределяющей формы в электродной реакции.
На основании вывода о природе аналитического сигнала в условиях катодной поляризации РПЭ проведены эксперименты по определению влияния предэлектролиза на величину катодного тока в растворах $\mathrm{Na}_{2} \mathrm{~S}_{2}$. В качестве потенциала предэлектролиза выбран $E_{3}=-0,5 \mathrm{~B}$, при котором сульфид- и полисульфид-ионы окисляются с образованием $\mathrm{HgS}$ (рис. 3, б). Такое значение потенциала предэлектролиза является оптимальным для перевода сульфид-ионов в $\mathrm{HgS}$ при определении сульфидов в щелочной среде [23]. Варьирование продолжительности предэлектролиза показало, что величина катодного тока при последующей катодной развертке потенциалов пропорциональна времени накопления (рис. 5, 6).
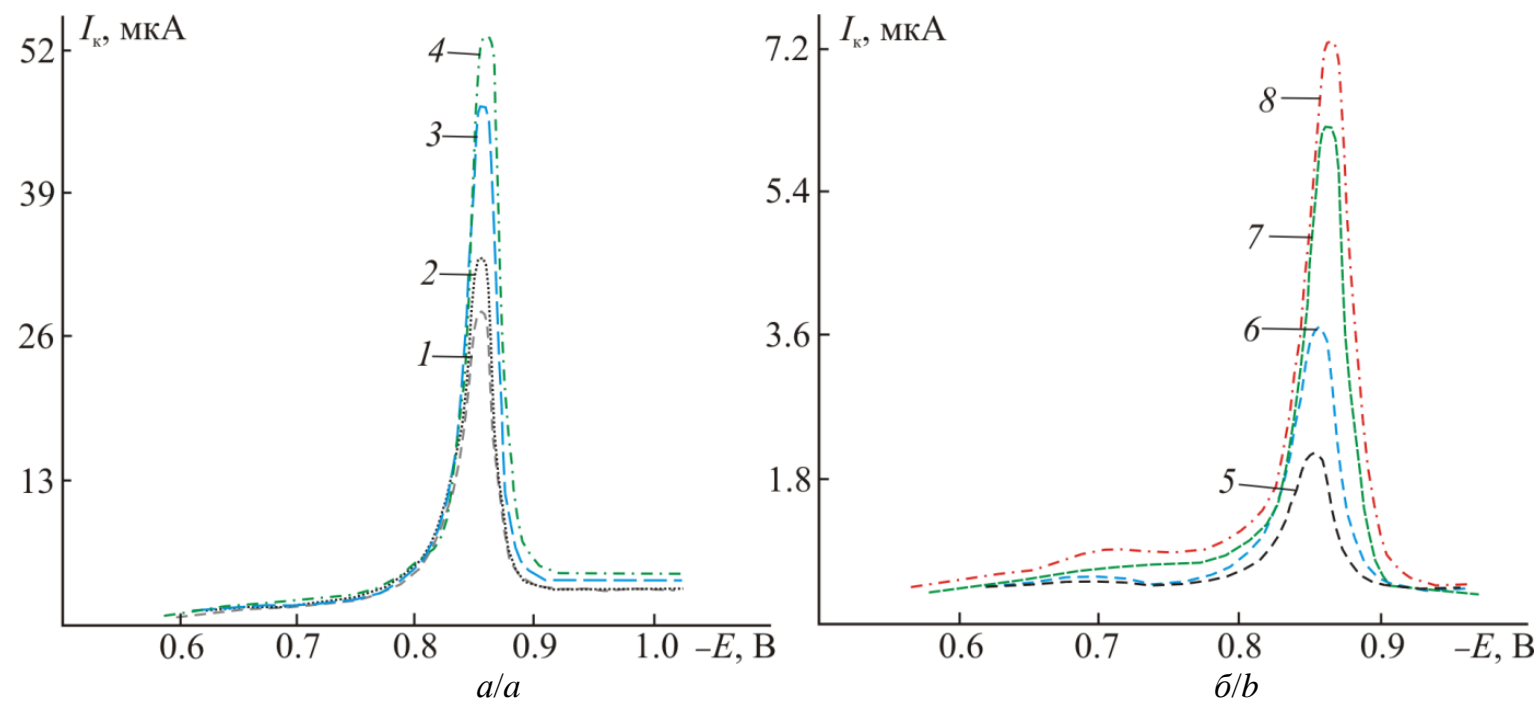

Рис. 5. Вольтамперограммы ртутно-пленочного электрода в растворе $4 \cdot 10^{-7} \mathrm{M} \mathrm{Na}_{2} \mathrm{~S}_{2}+0,1 \mathrm{M} \mathrm{NaOH}$ в условиях постояннотоковой (a) и переменнотоковой (б) катодной инверсионной вольтамперометрии при различной продолжстельности предэлектролиза при $E_{3}=-0,5$ B: 1) 30, 2) 60, 3) 120 , 4) 150, 5) 30, 6) 60, 7) 120 , 8) $150 \mathrm{c}$ $\left(E_{\text {нач }}=-0,5 \mathrm{~B} ; E_{\text {кон }}=-1,1 \mathrm{~B} ; v=40 \mathrm{MB} / \mathrm{c} ; \Delta U=3 \mathrm{MB} ; \theta=0^{\circ} ; f=25 \mathrm{\Gamma u}\right)$

Fig. 5. Voltammograms of $\mathrm{Hg}$-film electrode in solution $4 \cdot 10^{-7} \mathrm{M} \mathrm{Na}_{2} \mathrm{~S}_{2}+0,1 \mathrm{M} \mathrm{NaOH}$ under conditions of direct (a) and alternating current (b) cathodic stripping voltammetry at different duration of preelectrolysis at $\left.E_{\text {pre }}=-0,5 \mathrm{~V}: 1\right) 30$, 2) 60,3$) 120$, 4) 150,5$) 30,6) 60,7) 120,8) 150 \mathrm{~s}\left(E_{\text {rinit }}=-0,5 \mathrm{~V} ; E_{\text {end }}=-1,1 \mathrm{~V} ; v=40 \mathrm{mV} / \mathrm{s} ; \Delta U=3 \mathrm{mV} ; \theta=0^{\circ} ; f=25 \mathrm{~Hz}\right)$

При увеличении продолжительности предэлектролиза до 4-5 мин значение катодного тока становится практически постоянным, что связано, по-видимому, с образованием сплошного слоя $\mathrm{HgS}$ определенной толщины на поверхности электрода, при увеличении которого возрастания катодного тока не происходит. В зависимости от концентрации $\mathrm{S}_{2}{ }^{2-}$-ионов в растворе оптимальным временем предэлектролиза является 1-2 мин. В условиях катодной инверсионной вольтамперометрии концентрационная зависимость величины аналитического сигнала $\left(I_{\mathrm{K}}\right)$ является линейной в широком интервале концентраций дисульфид-ионов $1 \cdot 10^{-7}-1 \cdot 10^{-3} \mathrm{M}$ (рис. 6), что позволяет использовать такой метод в аналитических целях.

В отличие от $\mathrm{S}_{2}{ }^{2-}$-ионов, катодный процесс с участием трисульфид-ионов в условиях линейной поляризации РПЭ без предэлектролиза протекает при менее отрицательных потенциалах $E=-0,6 \ldots-0,8$ В (рис. 7). Наблюдаемое смещение потенциала $E_{\max }$ на $\sim 0,1$ В хорошо согласуется с расчетной зависимостью $E^{\circ}=f($ n) при возрастании степени полисульфидности n от 2 до 3 (рис. 1). Максимум катодного тока при этом характеризуется большей шириной по шкале потенциалов и более пологим начальным

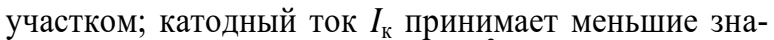
чения, чем в случае растворов $\mathrm{S}_{2}{ }^{2-}$-ионов с такой же концентрацией (рис. 4, 7). Наблюдаемая форма и величина максимума катодного тока свидетельствует об отличительных признаках процесса восстановления по сравнению с процессом в растворах $\mathrm{Na}_{2} \mathrm{~S}_{2}$ : смещение $E_{\max }$ в область положительных потенциалов и снижение $I_{\text {к }}$ свидетельствуют о преимущественном участии в электродном процессе ионной формы $\mathrm{S}_{3}{ }^{2-}$, а не $\mathrm{HgS}$. Концентрационная зависимость $I_{\text {к }}$ без использования предэлектролиза является линейной, как и в случае растворов $\mathrm{Na}_{2} \mathrm{~S}_{2}$. 

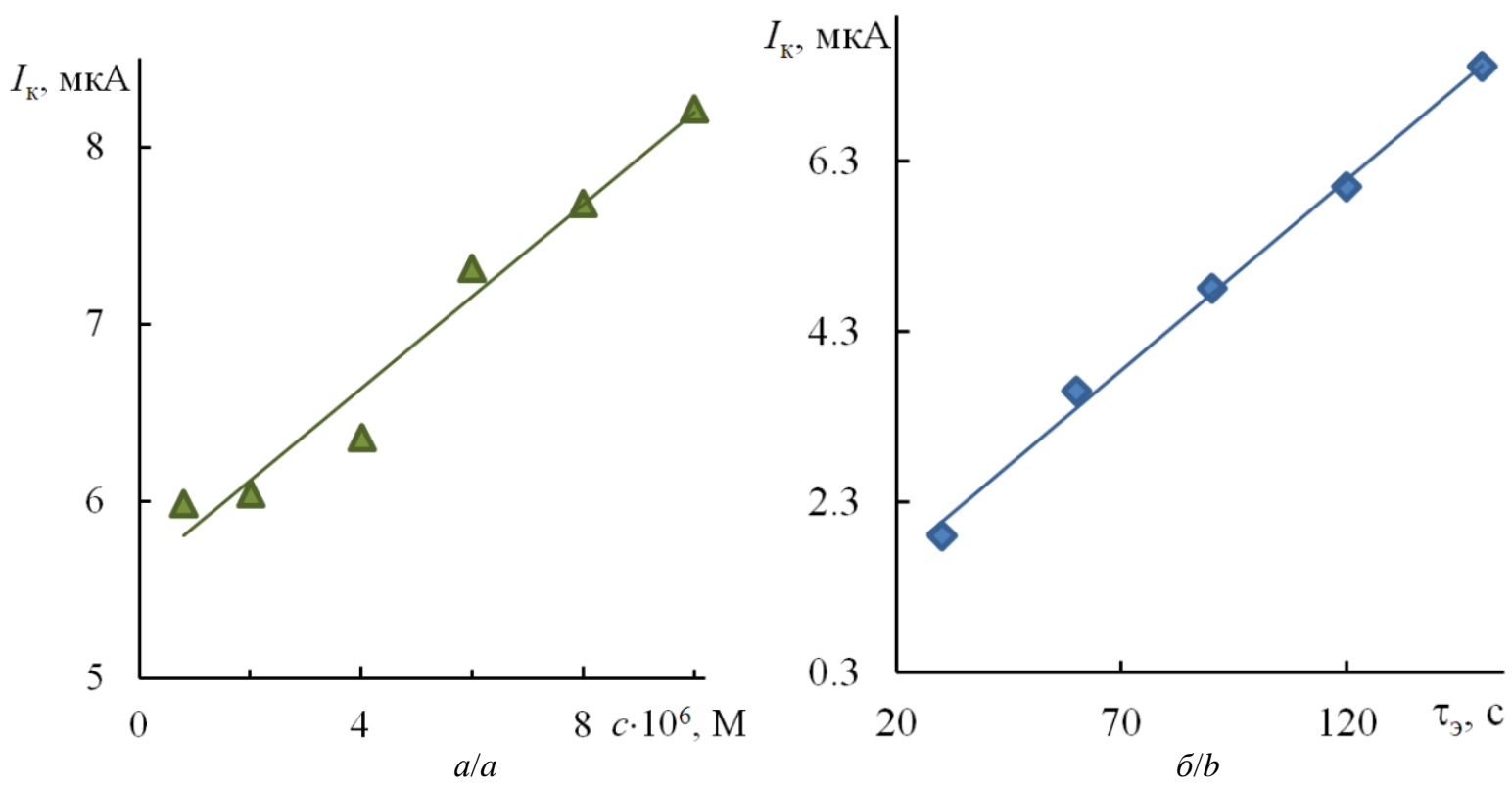

Pис. 6. Зависимости величины максимума катодного тока ртутно-пленочного электрода (а) от концентрации $\mathrm{Na}_{2} \mathrm{~S}_{2}$ (фон 0,1 M NaOH) без предэлектролиза и (б) от продолжительности предэлектролиза $\tau_{\ni}$ при $E_{\ni}=-0,5 \mathrm{~B}$ в растворе $4 \cdot 10^{-7} \mathrm{M} \mathrm{Na}_{2} \mathrm{~S}_{2}+0,1 \mathrm{M} \mathrm{NaOH}$ по данным переменнотоковой вольтамперометрии $\left(E_{\text {нач }}=-0,5 \mathrm{~B}\right.$; $\left.E_{\text {кон }}=-1,1 \mathrm{~B} ; \mathrm{v}=40 \mathrm{MB} / \mathrm{c} ; \Delta U=3 \mathrm{MB} ; \theta=0^{\circ} ; f=25 \Gamma \mathrm{u}\right)$

Fig. 6. Dependences of the peak currents of $\mathrm{Hg}$-film electrode (a) on concentration of $\mathrm{Na}_{2} \mathrm{~S}_{2}$ (supporting electrolyte $0,1 \mathrm{M}$ $\mathrm{NaOH}$ ) without preelectrolysis and (b) on duration of preelectrolysis $\tau_{3}$ at $E_{\text {pre }}=-0,5 \mathrm{~V}$ in solution $4 \cdot 10^{-7} \mathrm{M} \mathrm{Na}_{2} \mathrm{~S}_{2}+$ $0,1 \mathrm{M} \mathrm{NaOH}$ on alternating current voltammetry data $\left(E_{\text {init }}=-0,5 \mathrm{~V} ; E_{\text {end }}=-1,1 \mathrm{~V} ; \mathrm{v}=40 \mathrm{mV} / \mathrm{s} ; \Delta U=3 \mathrm{mV} ; \theta=0^{\circ}\right.$; $f=25 \mathrm{~Hz}$ )
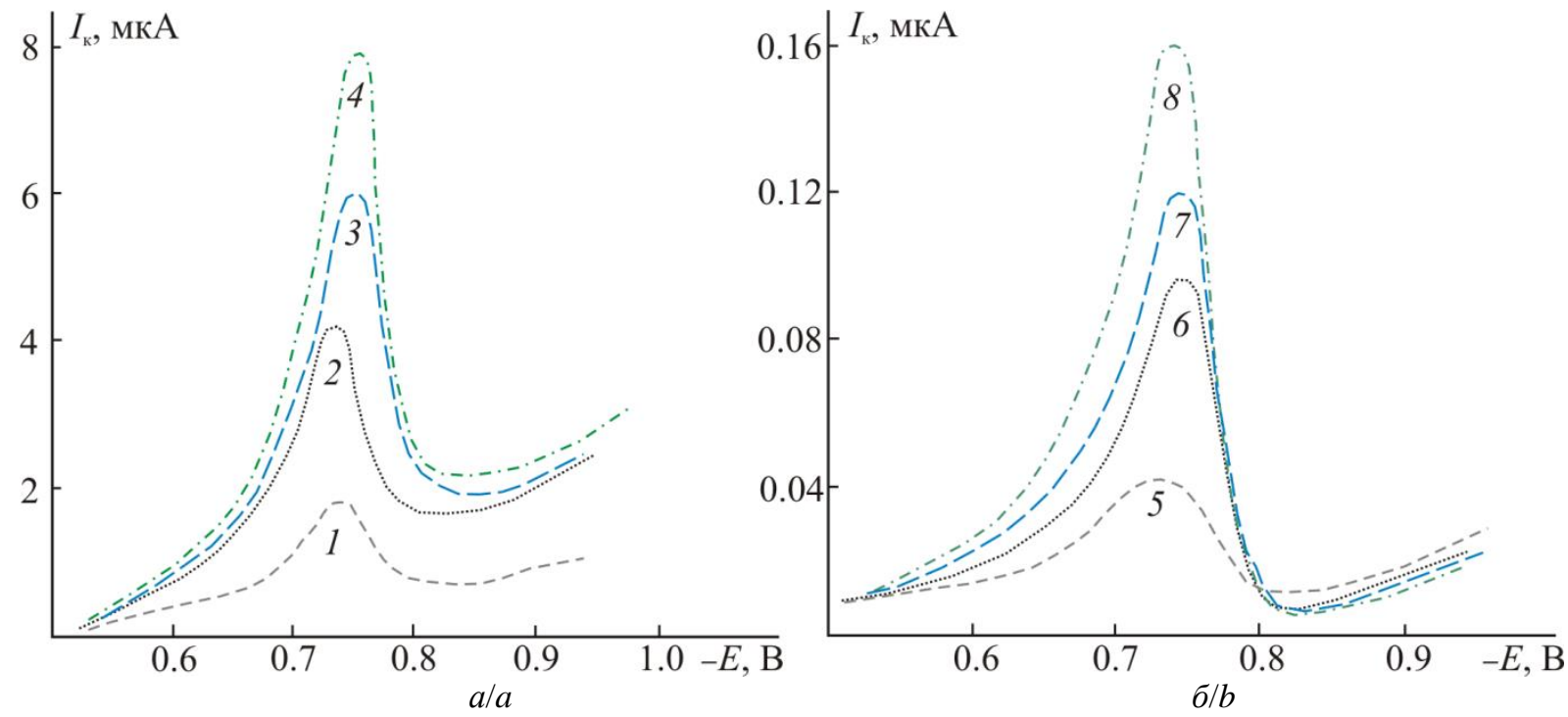

Рис. 7. Вольтамперограммы ртутно-пленочного электрода в растворах $\mathrm{Na}_{2} \mathrm{~S}_{3}+0,1 \mathrm{M} \mathrm{NaOH}$ в условиях линейной постояннотоковой (a) и переменнотоковой (б) вольтамперометрии при различных концентрациях $\mathrm{Na}_{2} \mathrm{~S}_{3}$ : 1) $4 \cdot 10^{-7}$, 2) $6 \cdot 10^{-7}$, 3) $8 \cdot 10^{-7}$, 4) $1 \cdot 10^{-6}$, 5) $2 \cdot 10^{-7}$, 6) $6 \cdot 10^{-7}$, 7) $8 \cdot 10^{-7}$, 8) $1 \cdot 10^{-6} \mathrm{M}\left(E_{\text {нач }}=-0,5 \mathrm{~B} ; E_{\text {кон }}=-1,1 \mathrm{~B}\right.$; $\left.v=40 \mathrm{MB} / \mathrm{c} ; \Delta U=3 \mathrm{mB} ; \theta=0^{\circ} ; f=25 \mathrm{\Gamma u}\right)$

Fig. 7. Voltammograms of $\mathrm{Hg}$-film electrode in solutions $\mathrm{Na}_{2} \mathrm{~S}_{3}+0,1 \mathrm{M} \mathrm{NaOH}$ under conditions of direct (a) and alternating current (b) voltammetry at different concentrations of $\mathrm{Na}_{2} \mathrm{~S}_{3}:$ 1) $4 \cdot 10^{-7}$, 2) $6 \cdot 10^{-7}$, 3) $8 \cdot 10^{-7}$, 4) $\left.1 \cdot 10^{-6}, 5\right) 2 \cdot 10^{-7}$, 6) $\left.\left.6 \cdot 10^{-7}, 7\right) 8 \cdot 10^{-7}, 8\right) 1 \cdot 10^{-6} M\left(E_{\text {init }}=-0,5 \mathrm{~V} ; E_{\text {end }}=-1,1 \mathrm{~V} ; v=40 \mathrm{mV} / \mathrm{s} ; \Delta U=3 \mathrm{mV} ; \theta=0^{\circ} ; f=25 \mathrm{~Hz}\right)$

Для установления возможности аналитического применения метода катодной ИВА с целью определения $\mathrm{S}_{3}{ }^{2-}$-ионов также использовали предварительный электролиз при $E_{\ni}=-0,5$ В. Как и в случае $S_{2}{ }^{2-}$-ионов, зависимость $I_{\text {к }}$ от продолжительности электролиза ли- нейна в широком временном интервале (рис. 8). При длительности предэлектролиза, превышающей 4-5 мин, зависимость $I_{\mathrm{K}}=f\left(\tau_{э}\right)$ выходит на насыщение вследствие формирования сплошной пленки продуктов окисления полисульфидов на поверхности электрода. 

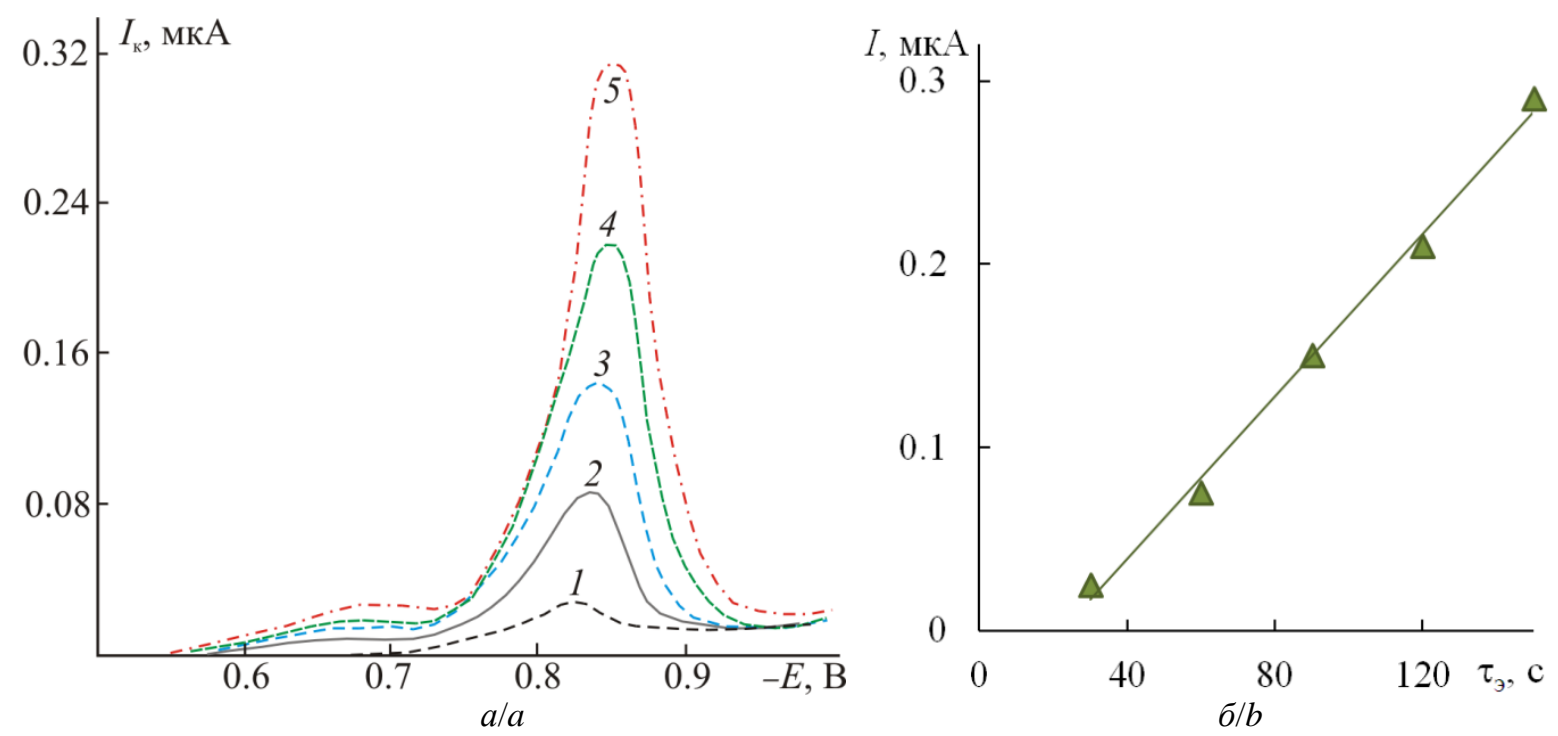

Pис. 8. Вольтамперограммы ртутно-пленочного электрода (а) в растворе $4 \cdot 10^{-8} \mathrm{M} \mathrm{Na}_{2} \mathrm{~S}_{3}+0,1 \mathrm{M} \mathrm{NaOH}$ в условиях переменнотоковой вольтамперометрии при различной продолжительности предэлектролиза при $E_{3}=-0,5 \mathrm{~B}$ : 1) 30,2$) 60,3) 90,4) 120,5) 150 \mathrm{c}$, и (б) зависимости величины максимума катодного тока от продолжительности предэлектролиза $\tau_{\ni}\left(E_{\text {нач }}=-0,5 \mathrm{~B} ; E_{\text {кон }}=-1,1 \mathrm{~B} ; \mathrm{v}=40 \mathrm{MB} / \mathrm{c} ; \Delta U=3 \mathrm{MB} ; \theta=0^{\circ} ; f=25\right.$ Ги)

Fig. 8. Voltammograms of $\mathrm{Hg}$-film electrode (a) in solution $4 \cdot 10^{-8} \mathrm{Na}_{2} \mathrm{~S}_{3}+0,1 \mathrm{M} \mathrm{NaOH}$ under conditions of alternating current voltammetry at different duration of preelectrolysis at $\left.\left.\left.\left.\left.E_{p r e}=-0,5 \mathrm{~V}: 1\right) 30,2\right) 60,3\right) 90,4\right) 120,5\right) 150 \mathrm{~s}$, and (b) dependences of cathodic peak current on duration of preelectrolysis $\tau_{\ni}\left(E_{\text {init }}=-0,5 \mathrm{~V} ; E_{\text {end }}=-1,1 \mathrm{~V} ; v=40 \mathrm{mV} / \mathrm{s}\right.$; $\Delta U=3 \mathrm{mV} ; \theta=0^{\circ} ; f=25 \mathrm{~Hz}$ )

Оптимальной продолжительностью предэлектролиза для получения величин $I_{\text {к }}$ достаточных для измерения аналитического сигнала, является 2-3 мин. Интересно отметить, что потенциал катодного максимума $E_{\max }$ при регистрации вольтамперограммы после предварительного электролиза смещен в область отрицательных потенциалов (рис. 8) по сравнению с экспериментом без использования предэлектролиза (рис. 7), что свидетельствует о преимущественном участии $\mathrm{HgS}$ в катодном процессе после предварительного формирования продуктов окисления полисульфидов. Результаты измерений величины аналитического сигнала с использованием предэлектролиза показали, что зависимость $I_{\mathrm{K}}=f\left[c\left(\mathrm{~S}_{3}{ }^{2-}\right)\right]$ является линейной в широком интервале концентраций $\mathrm{S}_{3}{ }^{2-}$-ионов $1 \cdot 10^{-7}-1 \cdot 10^{-3} \mathrm{M}$.

Особенностью зависимости $I_{\text {к }}$ от концентрации субстрата в растворах трисульфида является существенно меньшие, чем в растворах дисульфида, катодные токи как с использованием предэлектролиза, так и без него (рис. 4-8). При одинаковых молярных концентрациях $\mathrm{S}_{3}{ }^{2-}$-ионы содержат большее количество атомов серы, чем $\mathrm{S}_{2}{ }^{2-}$-ионы. При переходе всей серы из полисульфидной формы в $\mathrm{HgS}$ величина катодного тока должна увеличиваться в растворах трисульфида, а не уменьшаться, как это следует из экспериментальных данных. Следовательно, возрастание степени полисульфидности приводит к относительному уменьшению количества электроактивного промежуточного продукта, катодное восстановление которого приводит к появлению максимумов тока на вольтамперограммах. С учетом возможных электродных реакций окисления трисульфида на РПЭ в условиях предэлектролиза

$$
\begin{aligned}
\mathrm{S}_{3}^{2-}+\mathrm{Hg} & \rightarrow \mathrm{HgS}+2 \mathrm{~S}+2 \mathrm{e}, \\
\mathrm{S}_{3}^{2-} & \rightarrow 3 \mathrm{~S}+2 \mathrm{e},
\end{aligned}
$$

наиболее вероятной причиной уменьшения величины

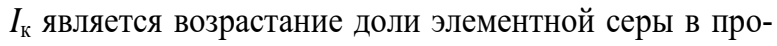
дуктах окисления при возрастании степени полисульфидности. За счет высокого электросопротивления элементной серы доля тока её восстановления в

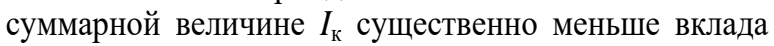
тока восстановления $\mathrm{HgS}$, обладающего электронной проводимостью. По-видимому, возрастание степени полисульфидности приводит к кинетическим затруднениям в образовании $\mathrm{HgS}$ за счет замедления процесса взаимодействия ртути с кластерами серы и, как следствие, к снижению величины катодного тока.

\section{Выводы}

1. На основе анализа литературных данных по термодинамическим свойствам полисульфидов предложены эмпирические уравнения для расчета изменения энергии Гиббса образования $\mathrm{S}_{n}{ }^{2-}$-ионов в водных растворах и их стандартных потенциалов в зависимости от степени полисульфидности в интервале $n=1 \ldots 8$. Уравнения позволяют уточнить характеристики $\mathrm{S}_{n}{ }^{2-}$-ионов и проводить расчеты зависимостей «состав-свойство» для равновесных многокомпонентных систем.

2. Проведены расчеты равновесных активностей ионно-молекулярных форм в системе $\mathrm{Hg}-\mathrm{S}-\mathrm{H}_{2} \mathrm{O}$ при различных значениях рН и потенциалов. Показано, что основными продуктами при окислении полисульфидов на ртутном электроде в щелочной среде $(\mathrm{pH}=13)$ независимо от состава исходного раствора являются $\mathrm{HgS}$ и $\mathrm{S}$, последующее катод- 
ное восстановление которых позволит количественно определить суммарное содержание сульфидной и полисульфидной форм в растворе и среднюю степень полисульфидности в ионах $\mathrm{S}_{n}{ }^{2-}$.

3. Установлены оптимальные условия определения полисульфид-ионов $\mathrm{S}_{2}{ }^{2-}$ и $\mathrm{S}_{3}{ }^{2-}$ в щелочной среде $(0,1 \mathrm{M} \mathrm{NaOH})$ на ртутно-пленочном электроде с использованием постоянно- и переменнотоковой катодной инверсионной вольтамперометрии: потенциал предэлектролиза $-0,5$ В (х. с. э.); продолжительность предэлектролиза $1 . .2$ мин; по-

\section{СПИСОК ЛИТЕРАТУРЫ}

1. Bajpai P. Biermann's handbook of pulp and paper: raw material and pulp making. - Amsterdam: Elsevier, 2018. - 668 p.

2. Технология обработки материалов / под. ред. В. Б. Лившица. М.: Юрайт, 2018. - 381 с.

3. Polysulfide polymers: synthesis, blending, nanocomposites, and applications / A. Pirayesh, M. Salami-Kalajahi, H. RoghaniMamaqani, F. Najafi // Polymer Reviews. - 2019. - V. 59. № 1. - P. 124-148.

4. Building materials in civil engineering / Ed. by H. Zhang. Cambridge: Woodhead Publ., 2011. - 440 p.

5. Metal-based nanostructured materials for advanced lithium-sulfur batteries / J. Balach, J. Linnemann, T. Jaumann, L. Giebeler // Journal of Materials Chemistry A. - 2018. - V. 6 . P. 23127-23168.

6. Recent trends on tailoring cathodes for room-temperature Na-S batteries / D. Kumar, D. Kanchan, S. Kumar, K. Mishra // Materials Science for Energy Technologies. - 2019. - V. 2. P. 117-129.

7. Distribution of polysulfide in human biological fluids and their association with amylase and sperm activities / M. Ikeda, Y. Ishima, V.T.G. Chuang et al. // Molecules. - 2019. - V. 24. P. 1689 (11)

8. Method for the determination of inorganic polysulfide distribution in aquatic systems / A. Kamyshny, I. Ekeltchik, J. Gun, O. Lev // Analytical Chemistry. - 2006. - V. 78. - P. 2631-2639.

9. Steudel R., Chivers T. The role of polysulfide dianions and radical anionsin the chemical, physical and biological sciences, including sulfur-based batteries // Chemical Society Reviews. - 2019. V. 48. - P. 3279-3319.

10. Bedoya-Lora F.E., Hankin A., Kelsall G.H. In situ determination of polysulfides in alkaline hydrogen sulfide solutions // Electrochimica Acta - 2019. - V. 314 - P. 40-48.

11. Determination of the reduced sulfur species in the water of anoxic basins / A.V. Dubinin, T.P. Demidova, M.N. Rimskaya-Korsakova, L.S. Semilova, O.A. Ocherednik // Physical Oceanography. 2019. - V. 26. - № 1. - P. 32-46.

12. Quantitative and qualitative determination of polysulfide species in the electrolyte of a lithium-sulfur battery using HPLC ESI/MS with one-step derivatization / D. Zheng, D. Qu, X.-Q. Yang, X. Yu, H.-S. Lee, D. Qu // Advanced Energy Materials. - 2015. - V. 5. P. 1401888 (1-5)

13. Exploiting XPS for the identification of sulfides and polysulfides / M. Fantauzzi, B. Elsener, D. Atzei, A. Rigoldi, A. Rossi // RSC Advances. - 2015. - V. 5. - P. 75953-75963.

14. Spectral monitoring of the formation and degradation of polysulfide ions inalkaline conditions / I. Filpponen, A. Guerra, A. Hai, L.A. Lucia, D.S. Argyropoulos // Industrial \& Engineering Chemistry Research. - 2006. - V. 45. - P. 7388-7392.

15. Le Guillanton G., Do Q. T., Elothmani D. Determination of mixtures of polysulfides by cyclic voltammetry // Journal of The Electrochemical Society. - 1996. - V. 143. - № 10. P. L223-L225.

16. Electroanalytical methods in characterization of sulfur species in aqueous environment / I. Ciglenečki, M. Marguš, E. Bura-Nakić, следующая катодная поляризация до $E_{\text {кон }}=-1,0 \mathrm{~B}$; предварительное деаэрирование растворов азотом. В этих условиях концентрационная зависимость величины максимума катодного тока $I_{\mathrm{K}}$ при $E_{\max }=-0,8 \ldots-0,9$ В линейна в интервале концентраций полисульфида $1 \cdot 10^{-7} \ldots 1 \cdot 10^{-3} \mathrm{M}$. Метод может быть использован для определения общего содержания полисульфидов в растворах и для расчета средней степени полисульфидности в ионах $\mathrm{S}_{n}{ }^{2-}$.

I. Milanović // Journal of Electrochemical Science and Engineering. - 2014. - V. 4. - № 4. - P. 155-163.

17. Kušan A.C., Frka S., Ciglenečki I. Electrochemical evidence of non-volatile reduced sulfur species in water-soluble fraction of fine marine aerosols // Atmosphere. - 2019. - V. 10. - P. 674 (1-14).

18. Kovacova Z., Zezula I. Polarographic reduction of polysulphides. 1. Reduction wave of disulphide // Collection of Czechoslovak Chemical Communications. - 1972. - V. 37. - № 6. - P. 935-941.

19. Kovacova Z., Zezula I. Reduction wave of the tetrasulphide anion // Collection of Czechoslovak Chemical Communications. 1974. - V. 39. - № 3. - P.722-728.

20. Rozan T.F., Theberge S.M., Luther G. Quantifying elemental (So) bisulfide (HS-) and polysulfides ( $\mathrm{Sn2}-$ ) using a voltammetric method // Analytica Chimica Acta. - 2000. - V. 415. - P. 175-184.

21. Determination of total ionic polysulfides by differential pulse polarography / S. Kariuki, M.J. Morra, K.J. Umiker, I.F. Cheng // Analytica Chimica Acta. - 2001. - V. 442. - P. 277-285.

22. Voltammetric investigation on sulfide ions in aqueous solutions with mercury-coated platinum microelectrodes / M.A. Baldo, S. Daniele, C. Bragato, G.A. Mazzocchin // Analytica Chimica Acta. - 2002. - V. 464. - P. 217-227.

23. Kovaleva S.V., Cheremukhina N.M., Gladyshev V.P. Voltammetric determination of sulfide ions // Journal of Analytical Chemistry. - 2004. - V. 59. - № 8. - P. 749-752.

24. ГОСТ 2053-77 Реактивы. Натрий сернистый 9-водный. Технические условия (с Изменениями № 1, 2). - М.: ИПК Изд-во стандартов, 2001. $-5 \mathrm{c}$.

25. Marony G., Valensi G. Function thermodynamiques standard des ions mono et polysulfures en solution aqueuese // International Committee of Electrochemical Thermodynamics and Kinetics. London: Butterworths Scientific publication, 1959. - P. 155-165.

26. Гладышев В.П., Дубинина Л.К. Свободная энергия образования анионов полиселенидов в водных растворах // Журнал физической химии. - 1982. - Т. 56. - Вып. 2. - С. 431-433.

27. Ковалева С.В., Гладышев В.П. Потенциалы полителлуридных систем // Журнал физической химии. - 1982. - Т. 56. - Вып. 9. C. 2339-2342.

28. Selected values of chemical thermodynamic properties. Tables for the first thirty-four elements in the standard order of arrangement. NDS Technical Note 270-3 / D.D. Wagman, W.H. Evans, I. Halow, V.B. Parker, S.M. Bailey, R.H. Schumm. - Washington: Institute for Basic Standards, 1968. - $264 \mathrm{p}$.

29. Ковалева С.В. Окислительно-восстановительные реакции с участием различных форм водорода, отрицательных ионов и гидридов Р- и S-элементов: автореферат дис. ... д-ра хим. наук. - Томск, 2000. $-42 \mathrm{c}$.

30. Ковалева С.В., Гладышев В.П., Черемухина Н.М. Электрохимическое поведение элементной серы и сульфида ртути на ртутно-пленочном электроде // Вестник Томского государственного педагогического университета. - 2003. - Т. 36. № 4. - C. 52-54.

Поступила 29.02.2020 2. 
Информация об авторах

Ковалева С.В., доктор химических наук, профессор кафедры химии и методики обучения химии биологохимического факультета Томского государственного педагогического университета.

Коршунов А.В., доктор химических наук, профессор отделения естественных наук Школы базовой инженерной подготовки Национального исследовательского Томского политехнического университета.

Аксиненко О.С., методист Управления образования Администрации Томского района.

Абрамова П.В., кандидат химических наук, ассистент отделения естественных наук Школы базовой инженерной подготовки Национального исследовательского Томского политехнического университета. 
UDC 546.222.4:543.552.054.1:544.6.076.32-034.791

\title{
DETERMINATION OF POLYSULFIDE IONS IN ALKALINE MEDIUM UNDER CONDITIONS OF CATHODIC STRIPPING VOLTAMMETRY WITH MERCURY-FILM ELECTRODE
}

\author{
Svetlana V. Kovaleva ${ }^{1}$, \\ svetkovaleva@rambler.ru
}

Andrey V. Korshunov², korshunov@tpu.ru

Olga S. Aksinenko3, crumba88@mail.ru

Polina V. Abramova², polya.bozhcko@yandex.ru

1 Tomsk State Pedagogical University, 60, Kievskaya street, Tomsk, 634061, Russia

2 National Research Tomsk Polytechnic University, 30, Lenin avenue, Tomsk, 634050, Russia

3 Department of Education, Tomsk District Administration, 2a, Kooperativny lane, Tomsk, 634009, Russia

Relevance. Polysulfides of alkaline, alkaline earth metals and ammonium are components of technological solutions in large-capacity industries (pulp and paper industry; blackening and bluing of metals; production of polysulfide elastomers), they are used to obtain new functional materials (sealants) and play a decisive role in functioning of chemical current sources of a new generation. In this regard, the study of the electrode processes involving polysulfides is relevant.

The aim of the research is to establish conditions for determining polysulfide ions in alkaline solutions using cathodic stripping voltammetry with a mercury-film electrode.

Objects: sodium polysulfides ( $\mathrm{Na}_{2} \mathrm{~S}_{2}, \mathrm{Na}_{2} \mathrm{~S}_{3}$ ) solutions, sodium hydroxide solutions.

Methods: direct and alternating current cathodic stripping voltammetry, cyclic voltammetry, accumulative electrolysis.

Results. The authors have proposed empirical equations to calculate the Gibbs energy of formation of $\mathrm{S}_{\mathrm{n}}{ }^{2-}$-ions in aqueous solutions and their standard potentials depending on the degree of polysulfidity in the range $n=1 \ldots 8$. The equilibrium activities of ion-molecular forms in the $\mathrm{Hg}-\mathrm{S}-\mathrm{H}_{2} \mathrm{O}$ system at different $\mathrm{pH}$ and potentials were calculated. The authors established the optimal conditions for determining polysulfide ions $\mathrm{S}_{2}{ }^{2-}$ and $\mathrm{S}_{3}{ }^{2-}$ in alkaline medium $(0,1 \mathrm{M} \mathrm{NaOH})$ at a mercury-film electrode using the direct and alternating current cathodic stripping voltammetry: preelectrolysis potential $-0,5 \mathrm{~V}$ (vs. Ag/AgCl); preelectrolysis duration $1 . . .2 \mathrm{~min}$; subsequent cathodic polarization to $E_{\text {end }}=-1,0 \mathrm{~V}$; preliminary deaeration of solutions with nitrogen. Under these conditions, the concentration dependence of the maximum cathodic current $I_{\text {cat }}$ at $E_{\max }=-0,8 \ldots-0,9 \mathrm{~V}$ is linear in the range of polysulfide concentrations $1 \cdot 10^{-7} \ldots 1 \cdot 10^{-3} \mathrm{M}$. The method can be used to determine the total content of polysulfides in solutions and to calculate the average degree of polysulfidity for $\mathrm{S}_{\mathrm{n}}{ }^{2-}$-ions.

\section{Key words:}

Polysulfide ions, alkaline electrolytes, direct and alternating current cathodic stripping voltammetry, cyclic voltammetry, mercury-film electrode, cathodic process.

\section{REFERENCES}

1. Bajpai P. Biermann's handbook of pulp and paper: raw material and pulp making. Amsterdam, Elsevier, 2018. 668 p.

2. Tekhnologiya obrabotki materialov [Technology of materials treatment]. Ed. by V.B. Livshits. Moscow, Yurait Publ. House, $2018.381 \mathrm{p}$.

3. Pirayesh A., Salami-Kalajahi M., Roghani-Mamaqani H., Najafi F. Polysulfide polymers: synthesis, blending, nanocomposites, and applications. Polymer Reviews, 2019, vol. 59, no. 1, pp. 124-148.

4. Building materials in civil engineering. Ed. by H. Zhang. Cambridge, Woodhead Publishing, 2011. $440 \mathrm{p}$.

5. Balach J., Linnemann J., Jaumann T., Giebeler L. Metal-based nanostructured materials for advanced lithium-sulfur batteries. Journal of Materials Chemistry A, 2018, vol. 6, pp. 23127-23168.

6. Kumar D., Kanchan D., Kumar S., Mishra K. Recent trends on tailoring cathodes for room-temperature Na-S batteries. Materials Science for Energy Technologies, 2019, vol. 2, pp. 117-129.

7. Ikeda M., Ishima Y., Chuang V. T. G. Distribution of polysulfide in human biological fluids and their association with amylase and sperm activities. Molecules, 2019, vol. 24, pp. 1689 (11).
8. Kamyshny A., Ekeltchik I., Gun J., Lev O. Method for the determination of inorganic polysulfide distribution in aquatic systems. Analytical Chemistry, 2006, vol. 78, pp. 2631-2639.

9. Steudel R., Chivers T. The role of polysulfide dianions and radical anionsin the chemical, physical and biological sciences, including sulfur-based batteries. Chemical Society Reviews, 2019, vol. 48, pp. 3279-3319.

10. Bedoya-Lora F.E., Hankin A., Kelsall G.H. In situ determination of polysulfides in alkaline hydrogen sulfide solutions. Electrochimica Acta, 2019, vol. 314, pp. 40-48.

11. Dubinin A.V., Demidova T.P., Rimskaya-Korsakova M.N., Semilova L.S., Ocherednik O.A. Determination of the reduced sulfur species in the water of anoxic basins. Physical Oceanography, 2019, vol. 26, no. 1, pp. 32-46.

12. Zheng D., Qu D., Yang X.-Q., Yu X., Lee H.-S., Qu D. Quantitative and qualitative determination of polysulfide species in the electrolyte of a lithium-sulfur battery using HPLC ESI/MS with one-step derivatization. Advanced Energy Materials, 2015, vol. 5, pp. $1401888(1-5)$

13. Fantauzzi M., Elsener B., Atzei D., Rigoldi A., Rossi A. Exploiting XPS for the identification of sulfides and polysulfides. RSC Advances, 2015, vol. 5, pp. 75953-75963. 
14. Filpponen I., Guerra A., Hai A., Lucia L. A., Argyropoulos D.S. Spectral monitoring of the formation and degradation of polysulfide ions inalkaline conditions. Industrial \& Engineering Chemistry Research, 2006, vol. 45, pp. 7388-7392.

15. Le Guillanton G., Do Q.T., Elothmani D. Determination of mixtures of polysulfides by cyclic voltammetry. Journal of the Electrochemical Society, 1996, vol. 143, no. 10, pp. L223-L225.

16. Ciglenečki I., Marguš M., Bura-Nakić E., Milanović I. Electroanalytical methods in characterization of sulfur species in aqueous environment. Journal of Electrochemical Science and Engineering, 2014, vol. 4, no. 4, pp. 155-163.

17. Kušan A.C., Frka S., Ciglenečki I. Electrochemical evidence of non-volatile reduced sulfur species in water-soluble fraction of fine marine aerosols. Atmosphere, 2019, vol. 10, pp. 674 (1-14).

18. Kovacova Z., Zezula I. Polarographic reduction of polysulphides. 1. Reduction wave of disulphide. Collection of Czechoslovak Chemical Communications, 1972, vol. 37, no. 6, pp.935-941.

19. Kovacova Z., Zezula I. Reduction wave of the tetrasulphide anion. Collection of Czechoslovak Chemical Communications, 1974, vol. 39, no. 3, pp.722-728.

20. Rozan T.F., Theberge S.M., Luther G. Quantifying elemental $\left(S^{0}\right)$ bisulfide $\left(\mathrm{HS}^{-}\right)$and polysulfides $\left(\mathrm{S}_{\mathrm{n}}{ }^{2-}\right)$ using a voltammetric method. Analytica Chimica Acta, 2000, vol. 415, pp. 175-184.

21. Kariuki S., Morra M.J., Umiker K.J., Cheng I.F. Determination of total ionic polysulfides by differential pulse polarography. Analytica Chimica Acta, 2001, vol. 442, pp. 277-285.

22. Baldo M.A., Daniele S., Bragato C., Mazzocchin G.A. Voltammetric investigation on sulfide ions in aqueous solutions with mercury-coated platinum microelectrodes. Analytica Chimica Acta, 2002, vol. 464, pp. 217-227.

23. Kovaleva S.V., Cheremukhina N.M., Gladyshev V.P. Voltammetric determination of sulfide ions. Journal of Analytical Chemistry, 2004, vol. 59, no. 8, pp. 749-752.

24. GOST 2053-77 Reactivy. Natriy sernisty 9-vodny. Tekhnicheskie usloviya (s Izmeneniyami no. 1, 2) [The Russ. State Standard
2053-77. Reactants. Sodium Sulfide 9-hydrate. Technical conditions (with Changes no. 1, 2)]. Moscow, Publ. House of Standards, 2001. 5 p.

25. Marony G., Valensi G. Function thermodynamiques standard des ions mono et polysulfures en solution aqueuese. International Committee of Electrochemical Thermodynamics and Kinetics. London, Butterworths Scientific publication, 1959. pp. 155-165.

26. Gladyshev V.P., Dubinina L.K. Svobodnaya energiya obrazovaniya anionov poliselenidov v vodnykh rastvorakh [Free energy of formation of polyselenide anions in aqueous solutions]. Russian Journal of Physical Chemistry, 1982, vol. 56, no. 2, pp. 431-433. In Rus.

27. Kovaleva S.V., Gladyshev V.P. Potentsialy politelluridnykh system [Potentials of polytelluride systems]. Russian Journal of Physical Chemistry, 1982, vol. 56, no. 9, pp. 2339-2342.

28. Wagman D.D., Evans W.H., Halow I., Parker V.B., Bailey S.M., Schumm R.H. Selected values of chemical thermodynamic properties. Tables for the first thirty-four elements in the standard order of arrangement. NDS Technical Note 270-3. Washington, Institute for Basic Standards, 1968. $264 \mathrm{p}$

29. Kovaleva S.V. Okislitelno-vosstanovitelnye reaktsii s uchastiem razlichnykh form vodoroda, otritsatelnykh ionov $i$ gidridiv $p$ - iselementov. Avtoreferat. Dis. Dokt. Nauk [Oxidation-reduction reactions with participation of different species of hydrogen, of negatively charged ions and of hydrides of p- and s-elements. Dr. Diss. Abstract]. Tomsk, 2000. $42 \mathrm{p}$.

30. Kovaleva S.V., Gladyshev V.P., Cheremukhina N.M. Elektrokhimicheskoe povedenie elementnoy sery i sulfida rtuti na rtutno-plenochnom elektrode [Electrochemical behavior of sulfur and mercury sulfide on Hg-film electrode]. Bulletin of Tomsk State Pedagogical University, 2003, vol. 36, no. 4, pp. 52-54. In Rus.

Received: 29 February 2020.

\section{Information about the authors}

Svetlana V. Kovaleva, Dr. Sc., professor, Tomsk State Pedagogical University.

Andrey V. Korshunov, Dr. Sc., professor, National Research Tomsk Polytechnic University.

Olga S. Aksinenko, methodologist, Department of Education, Tomsk District Administration.

Polina V. Abramova, Cand. Sc., assistant, National Research Tomsk Polytechnic University. 